\title{
Computationally Guided Assembly of Oriented Nanocubes by Modulating Grafted Polymer-Surface Interactions
}

\author{
Kargal. L. Gurunatha, Sarrah Marvi, Gaurav Arya, and Andrea R. Tao* \\ NanoEngineering Department, University of California, San Diego, 9500 Gilman Drive MC 0448, La Jolla, California 92093-0448, \\ United States
}

\section{Supporting Information}

ABSTRACT: The bottom-up fabrication of ordered and oriented colloidal nanoparticle assemblies is critical for engineering functional nanomaterials beyond conventional polymer-particle composites. Here, we probe the influence of polymer surface ligands on the self-orientation of shaped metal nanoparticles for the formation of nanojunctions. We examine how polymer graft-surface interactions dictate Ag nanocube orientation into either edge-edge or face-face nanojunctions. Specifically, we investigate the effect of end-functionalized polymer grafts on nanocube assembly outcomes, such as interparticle angle and interparticle distance. Our assembly results can be directly mapped onto our theoretical phase diagrams for nanocube orientation, enabling correlation of experimental variables (such as graft length and metal binding strength) with computational parameters. These results represent an important step toward unifying modeling and experimental approaches to understanding nanoparticle-polymer self-assembly.

KEYWORDS: Self-assembly, nanoparticle, course-grain model, polymer graft, nanocube, self-orientation, predictive assembly

A nisotropic nanoparticles often display unique size- and shape-dependent properties that are advantageous in technologies ranging from biosensing, ${ }^{1-3}$ to spectroscopy ${ }^{4,5}$ and optics, ${ }^{6}$ to solar cells. ${ }^{7}$ These shaped nanoparticles can also display unique collective properties when assembled into oligomeric architectures. In plasmonics, shaped metal nanoparticles can be assembled to form nanojunctions that exhibit intense electromagnetic hotspots ${ }^{8}$ or organized into large-area (or volume) assemblies for the formation of optical metamaterials. ${ }^{9}$ A major challenge is the assembly of anisotropic nanoparticles into targeted, functional orientations. Several experimental and theoretical advances have been made in the areas of DNA-programmable assembly ${ }^{10}$ and the crystallization of shaped, hard particles. ${ }^{11}$ However, these strategies can be difficult to implement for practical nanomaterials integration because of stringent assembly conditions on parameters such as solvent ionic strength, nanoparticle density, and total assembly volumes.

Nanoparticles incorporated into polymers are an especially attractive strategy for self-assembly as they allow for facile device integration using techniques that take advantage of batch, low-cost processing and enable the fabrication of stateof-the-art nanocomposite materials. The ability to direct the organization of nanoparticle assemblies by tuning the molecular-level interactions of the nanoparticle and polymer is a powerful tool for fabrication of plasmonic structures and other functional nanomaterials. Encapsulating shaped nanoparticles into polymers has the potential to harness the rich phase behavior exhibited by a multicomponent system of nanoparticles, nanoparticle grafts, and matrix polymer. Nonspecific interactions arising from van der Waals and steric forces can be engineered to give hierarchical, tunable, and dynamic nanoparticle structures. $^{12}$ For example, cooperative selfassembly of nanoparticles with diblock copolymers has been demonstrated to provide control of dipole-dipole and depletion forces of these mixed polymer-nanoparticles systems. ${ }^{13,14}$ Nanorods assembled with block copolymer grafts $^{15,16}$ have been able to achieve spherical, cylindrical, and lamellar microdomains and end-to-end nanorod alignment in polymer thin-films. The resulting morphologies of these polymer-nanoparticle composites are typically controlled by modulating the supramolecular framework of the block copolymer component.

In addition, a large body of theoretical and simulation work has greatly improved our understanding of nanoparticle assembly in various media like polymers. Studies employing integral equation theory, density functional theory, Monte Carlo, and molecular dynamics simulations have examined the interactions between nanoparticles within polymer melts ${ }^{17-20}$ and their modulation via polymer grafting. ${ }^{21-23}$ Simulations have begun to reveal the phase behavior of nanoparticles grafted with polymers, demonstrating how these building blocks self-assemble into phases reminiscent of block copolymer microphases. $^{24-27}$ However, few studies have been successful in relating computational parameters with experimental variables toward predicting nanoparticle assembly outcomes.

Recently, we and others have demonstrated through both experiments and modeling that the properties of the grafted polymer chains at the nanoparticle surface plays a crucial role in

Received: July 10, 2015

Revised: October 1, 2015 
determining the orientation of colloidal nanocubes in polymer matrix. ${ }^{9,28}$ In our previous work, we investigated the assembly of $\mathrm{Ag}$ nanocubes into two distinct orientations: face-face (FF) and edge-edge (EE). ${ }^{9}$ Using coarse-grained models and Monte Carlo simulations to calculate interparticle interactions, we examined the effect of grafting the nanocubes with polymer chains of varying length $(L)$ and varying polymer-surface interactions $\left(\varepsilon_{\mathrm{PS}}\right)$ in determining nanocube orientation. These results showed that a transition from $\mathrm{FF}$ to $\mathrm{EE}$ orientation occurred when $L$ is increased and when $\varepsilon_{\mathrm{PS}}$ is decreased. These computation results were also used to generate a nanocube orientation "phase" diagram. In our assembly experiments, we showed that nanocubes grafted with sufficiently long polymer grafts adopt EE orientations, which minimizes steric repulsion between polymer grafts as nanocubes approach each other. However, our experimental results provided only qualitative demonstration of the EE-FF phase transition and did not explicitly examine the effect of $\varepsilon_{\mathrm{PS}}$ on nanocube assembly outcomes.

In this work, we propose to directly correlate our previously reported nanocube orientation phase diagram with practical experimental parameters. First, we carry out assembly experiments to investigate the effect of polymer-surface interactions $\left(\varepsilon_{\mathrm{PS}}\right)$ on the orientation of self-assembled $\mathrm{Ag}$ nanocubes by comparing the assembly outcomes for end-functionalized polymer grafts that possess different interaction potentials with metal surfaces. In order to determine the critical $L$ for the EE-to-FF transition, we then carry out assembly experiments by varying the length of these polymer grafts within a smaller window of $L$ values than we previously tested. The ability to directly map these modeled parameters ( $L$ and $\varepsilon_{\mathrm{PS}}$ ) to experimental parameters (polymer molecular weight and chemical functionality) would bridge the divide between modeling and experimental approaches to understanding selfassembly, providing new insight into how molecular interactions govern global materials properties.

Experimental Methods. Materials. Silver nitrate $\left(\mathrm{AgNO}_{3}\right)$, copper chloride $\left(\mathrm{CuCl}_{2}\right)$, and polyvinylpyrrolidone (PVP $\left.\mathrm{M}_{\mathrm{W}}=55000\right)$ were purchased from Sigma-Aldrich and used as received. 1,5-Pentane-diol was purchased from Fluka. Polystyrene $\left(\mathrm{M}_{\mathrm{W}}=11900\right)$ was purchased from Polymer Source and mPEG thiol, PEG dithiol, and thiol PEG amine of molecular weight $1 \mathrm{k}, 2 \mathrm{k}$, and $5 \mathrm{k}$ were purchased from Nanocs Chemicals. Analytical grade solvents were used throughout the reaction.

Ag Nanocube Synthesis. Ag nanocubes were synthesized by previously reported protocol. ${ }^{9}$ The metal precursor solution was prepared by dissolving $0.20 \mathrm{~g} \mathrm{AgNO}_{3}$ with $40 \mu \mathrm{L} 0.043 \mathrm{M}$ $\mathrm{CuCl}_{2}$ in $5 \mathrm{~mL}$ of 1,5-pentanediol. Alternatively $0.10 \mathrm{~g}$ of polyvinylpyrrolidone (PVP) was dissolved in $5 \mathrm{~mL}$ of pentanediol. Twenty milliliters of pentanediol solution was added to a $100 \mathrm{~mL}$ round bottomed flask, and the solution temperature was maintained at $193{ }^{\circ} \mathrm{C}$ with continuous stirring. Next the precursor solutions with $\mathrm{AgNO}_{3}$ and PVP were injected into the hot pentanediol at a rate of $500 \mu \mathrm{L} / \mathrm{min}$ and $320 \mu \mathrm{L} / 30 \mathrm{~s}$, respectively. The injections were continued until the solution turned to an opaque yellow color. As synthesized nanocrystals were then characterized by UV-vis spectroscopy (Agilent Chemstation 8453). Purification of nanocrystals was carried out by diluting the original solution to $50 \mathrm{~mL}$ using absolute ethanol and centrifugation at $2500 \mathrm{rpm}$ for $10 \mathrm{~min}$. The supernatant was isolated and further diluted to a 1:2 mixture of deionized water and ethanol to obtain an approximate total volume of $150 \mathrm{~mL}$. This solution was subjected for the filtration with decreasing membrane pore sizes $(0.65,0.40$, and $0.22 \mu \mathrm{m})$. The final solution was centrifuged at $3400 \mathrm{rpm}$ for $60 \mathrm{~min}$ and redispersed with ethanol for the desired concentration. The extinction and scanning electron microscopy (SEM) revealed that the as-made nanocubes were highly monodispersed and $73 \pm 1.6 \mathrm{~nm}$ in size.

Nanocomposite Fabrication. Piranha cleaned silicon substrates were treated with hexamethyldisilazane vapor under vacuum at room temperature for $3 \mathrm{~h}$ to obtain hydrophobic surface. Polystyrene (3.5 wt \% of $11 \mathrm{k}$ ) was dissolved in toluene solution and filtered through $0.02 \mu \mathrm{m}$ pore size filter membrane. A $270 \mathrm{~nm}$ thick polystyrene thin film on the substrate was obtained by two-step spinning at $800 \mathrm{rpm}$ for $3 \mathrm{~s}$ and $1700 \mathrm{rpm}$ for $30 \mathrm{~s}$ (WS-400A-6NPP/LITE Laurell Technology Corp).

To prepare Ag nanocube films, $2 \mathrm{~mL}$ of nanocrystal solution was washed three times in ethanol at $3400 \mathrm{rpm}$ for $15 \mathrm{~min}$, and then residue was resuspended in $200 \mu \mathrm{L}$ chloroform $\left(\mathrm{CHCl}_{3}\right)$. A clean glass Petri dish washed thoroughly and filled with water at convex meniscus. Nanocrystal solution was then carefully spread over air-water interface, and then monolayer was left for $15 \mathrm{~min}$ to allow for the evaporation of organic solvent and equilibration. Polystyrene coated silicon wafers were then dip coated to make nanocube composite film. The surface coverage of individual and assembled nanocube was determined by Automated Quantitative Image Analysis software ${ }^{29}$ and ImageJ software by using SEM images.

Nanocube Assembly. The surface modification of PVP stabilized nanocubes was carried out by incubating the composite film in $1.5 \mathrm{mM}$ ethanol solution of bifunctional ligands at room temperature for $15 \mathrm{~h}$. For the long chain ligand (5k), a mixture of ethanol-methanol solution (2:1) was used. These substrates were then washed thoroughly with ethanol and water in order to remove excess ligand that was loosely bound to the PS film and dried with $\mathrm{N}_{2}$. Ligand exchange was confirmed by Raman spectroscopy with respective peaks corresponding to the ligands. Solvent annealing was carried out by exposing the substrates with $\mathrm{CHCl}_{3}$ vapor in a closed container, and samples were taken out and monitored through SEM at different time intervals to follow the assembly process.

Sample Characterization. Raman measurement of the samples was performed on Renishaw micro-Raman spectrometer (Renishaw Invia) coupled with Leica microscope with $50 \times$ objective (Leica N-plan) in the range of $200-1800 \mathrm{~cm}^{-1}$. A wavelength of $514 \mathrm{~nm}$ was used as an excitation source generated by $50 \mathrm{~mW}$ Ar-Ion LASER. The backscattered signal was collected on a Peltier-cooled $\left(-70^{\circ} \mathrm{C}\right) \mathrm{CCD}$ detector with $30 \mathrm{~s}$ acquisition time. Scanning electron microscopy (SEM) characterization was carried out using FEI UHR field emission SEM equipped with field emission cathode with a lateral resolution approximately $2 \mathrm{~nm}$. The accelerating voltage was operated between 5 and $15 \mathrm{kV}$.

Analysis of Nanocube Orientation and Interparticle Distance. Statistical analysis of nanocube orientation angle and interparticle distance (center-center) were determined with the help of ImageJ software. Annealing experiments were carried out until the films were stable, and with increasing annealing, time dewetting of the film occurred. Composite nanocube films with approximately the same density on different substrates were prepared and annealed after the ligand exchange at different time intervals. An area of $25 \mu \mathrm{m}^{2}$ was analyzed on SEM images to obtain angular statistics. 
Only nanocubes with interparticle distance below $25 \mathrm{~nm}$ were considered while analyzing the angle between two faceface and edge-edge oriented nanocubes. The inter cube angle $\theta=15^{\circ}$ was considered as a cutoff angle for EE structure, and below $\theta=15^{\circ}$ was considered as FF orientation of the cubes. For instances where cube-cube orientations angles were below $5^{\circ}$, exact angles were difficult to measure, and these orientations were considered as FF. Average orientation angle $\theta$ was obtained by Gaussian fitting of the statistical data, and in the case of PEG-DT (1k), PEG-Am (1k) induced assembly weighted average orientation angle was taken as the statistical data following exponential fit.

Potential of Mean Force Calculations. To investigate the role of the length $L$ and interactions $\varepsilon_{\mathrm{PS}}$ of polymer grafts in governing the relative orientation of the nanocubes in the selfassembled structures, we computed the potential of mean force (PMF) $W(d, \theta)$ between two polymer-grafted nanocubes (with an $80 \mathrm{~nm}$ edge length) as a function of their center-to-center separation distance $d$ and relative orientation $\theta$. Since the PMF essentially represents the free energy of interactions between nanocubes, its magnitude provides direct estimates of the probability $p(d, \theta) \propto \exp \left(-W(d, \theta) / k_{\mathrm{B}} T\right)$ of observing two nanocubes in a specific configuration $d$ and $\theta$, where $k_{\mathrm{B}}$ is the Boltzmann factor and $T$ is the temperature. The PMF profiles were computed for nine different combinations of $\left(L, \varepsilon_{\mathrm{PS}}\right)$ values by using an approach involving Monte Carlo simulations of coarse-grained models of the polymer-grafted nanocubes, as described earlier. ${ }^{9}$

Results and Discussion. In order to investigate the effects of $\varepsilon_{\mathrm{PS}}$ nanocube assembly, we grafted $\mathrm{Ag}$ nanocubes with bifunctional polyethylene glycol (PEG) grafts, where one end is terminated with a thiol group to anchor to the Ag surface and the other end is terminated with a chemical group that determines $\varepsilon_{\mathrm{PS}}$ (Figure $1 \mathrm{~B}$ ). We chose PEG-thiols that are end-
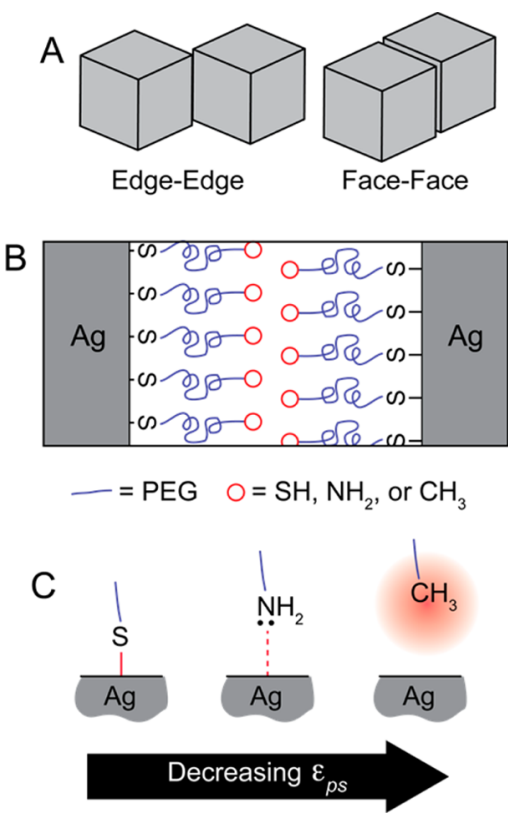

Figure 1. Schematic diagram showing (A) self-assembled Ag nanocubes in the face-face and edge-edge orientations and (B) self-assembled monolayer of bifunctional ligand on silver nanocube with different terminal group and PEG backbone. (C) The decreasing order of effective strength of attraction of polymer to the surface of nanocube is shown. terminated with one of the following: $-\mathrm{CH}_{3}$ (mPEG), $-\mathrm{NH}_{2}$ (PEG-Am), or -SH (PEG-DT). These bifunctional ligands were chosen because they are expected to possess different binding affinities toward metal surfaces and thus vastly different $\varepsilon_{\mathrm{PS}}$ values (Figure $1 \mathrm{C}$ ). For example, PEG-DT is expected to possess the highest $\varepsilon_{\mathrm{PS}}$ since thiols are known to bind strongly and covalently to Ag nanoparticles. ${ }^{30}$ PEG-Am is expected to possess only a moderate affinity to Ag nanoparticles (due to the formation of a dative bond), ${ }^{31,32}$ and $\mathrm{mPEG}$ is expected to display no affinity. Advantageously, these ligands are also commonly used as nanoparticle capping agents and are available in a range of molecular weights $\left(\mathrm{M}_{\mathrm{W}}=1 \mathrm{k}, 2 \mathrm{k}\right.$, and $5 \mathrm{k}$ ), which is useful for determining a critical $L$ for the EE-to-FF phase transition.

PEGylation of the Ag nanocube surface is achieved by incubating the nanocubes in a solution of the desired bifunctional PEG graft. First, the as-made cubes are deposited onto a thin-film of polystyrene (PS) using the method previously described. ${ }^{1}$ The PS-supported nanocubes are then incubated in a $1.5 \mathrm{mM}$ (solvent) solution of the PEG grafts for $15 \mathrm{~h}$. Polymer graft concentrations and incubation times were optimized to ensure that polyvinylpyrrolidone (PVP, the capping agent used in nanocube synthesis) is displaced and that the PEG grafts cover the majority of the nanocube surface. Raman spectra of the incubated nanocubes (Supporting Information, S1) confirm that PVP is removed and that the nanocube surface is effectively PEGylated. Raman peaks for polystyrene at $1001,1035,1360$, and $1583 \mathrm{~cm}^{-1}$ are present in every nanocube sample. ${ }^{33,34}$ For as-made nanocubes (black line), the adsorption of PVP is clearly identified by the intense peak that appears at $1670 \mathrm{~cm}^{-1}$, corresponding to the $\mathrm{C}=\mathrm{O}$ and $\mathrm{C}-\mathrm{N}$ (amide) stretching vibrations of PVP. ${ }^{35}$ This peak is not present in the nanocube films that have undergone PEGylation. Instead, the PEGylated nanocubes show distinct Raman bands at $1072 \mathrm{~cm}^{-1}$ corresponding to the $\mathrm{C}-\mathrm{O}$ stretch of the grafted PEG chains. ${ }^{36,37}$ For nanocubes modified with PEG-DT, the peak at $868 \mathrm{~cm}^{-1}$ is attributed to the $\beta_{\mathrm{CSH}}$ bend from the terminal $-\mathrm{SH}$ group. ${ }^{38}$ For nanocubes modified with PEG-Am, we also observe the weak vibrational band corresponding to the $\mathrm{NH}_{2}$ wag at $819 \mathrm{~cm}^{-1}$, whereas the Raman band corresponding to $\mathrm{NH}_{2}$ deformation overlaps with the Raman bands for PS in the range of $1580-1590 \mathrm{~cm}^{-1} .39$ Analysis of Raman spectra also suggests that changing the length of the polymer graft does not affect the displacement of PVP and subsequent PEGylation of the Ag nanocube surface (Supporting Information S2, S3, and S4).

The PEGylated nanocube films were then exposed to chloroform $\left(\mathrm{CHCl}_{3}\right)$ vapor to induce assembly and selforientation within the PS thin-film. ${ }^{9}$ Figure 2 shows representative scanning electron microscope (SEM) images of the nanocube assemblies after $180-240 \mathrm{~min}$ of solvent annealing. Because these SEM images reveal a heterogeneous mixture of $\mathrm{EE}$ and $\mathrm{FF}$ orientations, we analyzed nanocube orientation by determining the angle for each nanojunction generated by assembly. The angle between two adjacent cubes $(\theta)$ can vary from $0^{\circ}$ (corresponding to a perfect $\mathrm{FF}$ nanojunction) to $90^{\circ}$ (corresponding to a perfect $\mathrm{EE}$ nanojunction). Figure 3 shows histograms of $\theta$ for nanocubes modified with each of the three bifunctional PEG grafts (mPEG, PEG-Am, and PEG-DT) and three different PEG chain lengths $\left(M_{W}=1 k, 2 k\right.$, and $\left.5 k\right)$. For nanocubes modified with $\mathrm{mPEG}$, we observe that the nanocubes form nanojunctions with an average of $\theta=10.6 \pm 9.8^{\circ}$ for a $M_{W}=1 \mathrm{k}$. A 
A
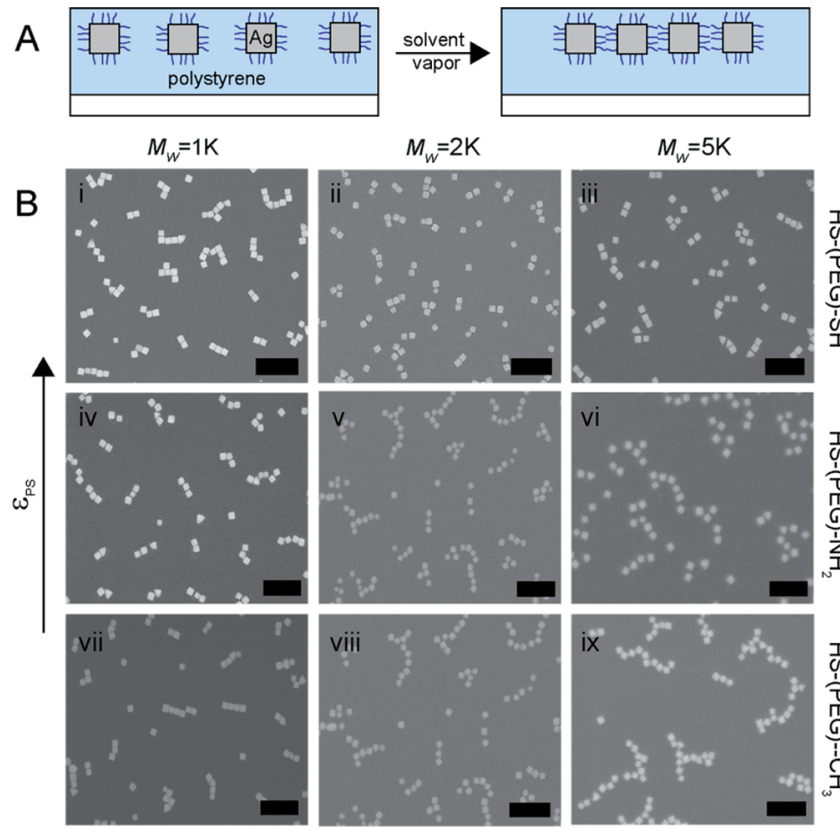

PEG Molecular Weight

Figure 2. (A) Schematic of bifunctional ligand modified Ag nanocube undergoing phase segregation upon annealing. (B) Scanning electron microscope images of PEG dithiol (i, ii, iii), thiol PEG amine (iv, v, vi), and mPEG thiol (vii, viii, ix) of different chain length induced selfassembly of nanocube (scale bar $500 \mathrm{~nm}$ ).

more heterogeneous distribution of angles is observed for longer PEG chains, with $\theta=50.1 \pm 48.2^{\circ}$ and $43.2 \pm 19.7^{\circ}$ for $\mathrm{M}_{\mathrm{W}}=2 \mathrm{k}$ and $5 \mathrm{k}$, respectively. For nanocubes modified with PEG-Am, we observe distribution of angles for all nanocube assemblies, with an average $\theta=13.5 \pm 0.4^{\circ}, 41.1 \pm 16^{\circ}$, and $41.3 \pm 18.3^{\circ}$ for $\mathrm{M}_{\mathrm{W}}=1 \mathrm{k}, 2 \mathrm{k}$, and $5 \mathrm{k}$, respectively. For nanocubes modified with PEG-DT, we observe that the nanocubes form nanojunctions with an average nanojunction angle $<15^{\circ}$, where $\theta=7.2 \pm 9.1^{\circ}, 8.48 \pm 16.6^{\circ}$, and $6.09 \pm 17^{\circ}$ for $\mathrm{M}_{\mathrm{W}}=1 \mathrm{k}, 2 \mathrm{k}$, and $5 \mathrm{k}$, respectively.

To map our assembly experiments onto our modeled phase diagram, we determined overall nanocube orientations as FF when the average nanojunctions angle is $\theta<15^{\circ}$ and $\mathrm{EE}$ when the average nanojunctions angle is $\theta>15^{\circ}$. Figure 4A shows our experimental phase diagram using these designations. For comparison, Figure 4B shows our predicted phase diagram based on the computed potential of mean force (PMF) profiles for purely EE- and FF-oriented nanocubes at nine different combinations of $L$ and $\varepsilon_{\mathrm{PS}}$ values, as derived in our previous work. ${ }^{9}$ The PMFs provide the distance-dependent free energy of interactions between two nanocubes, and the most favorable orientation of the nanocubes for each $L-\varepsilon_{\mathrm{PS}}$ combination is determined by selecting the energy curve that possesses the lowest energy minimum. Figure $4 \mathrm{c}$ shows the PMFs for three $L-\varepsilon_{\mathrm{PS}}$ combinations: for $L=4,8$, and 12 and $\varepsilon_{\mathrm{PS}}=0.4$.

Overall, our experimental and theoretical phase diagrams are consistent with each other in the prediction of a transition from FF to EE nanocube orientation for longer graft chains and lower graft-to-metal affinity. Based on our experimental data, we can determine that the critical $L$ for this transition is approximately $7 \mathrm{~nm}$, which corresponds to the calculated rodcoil length for a PEG chain with a $\mathrm{M}_{\mathrm{W}}=2 \mathrm{k}$. We also observe that nanocube assemblies, which possess a larger distribution of nanojunction angles, occur nearer to the FF to EE transition. This can be understood by examining the PMF curves for these particular $L-\varepsilon_{\mathrm{PS}}$ combinations that lie close to the transition line. For example, for the combination of $L=8$ and $\varepsilon_{\mathrm{PS}}=0.4$, the global energy minima for the FF and $\mathrm{EE}$ orientations are fairly close in energy. It is likely that the PMF curves look similar for the range of nanojunction angle between these two orientations, resulting in a broad distribution of orientations.

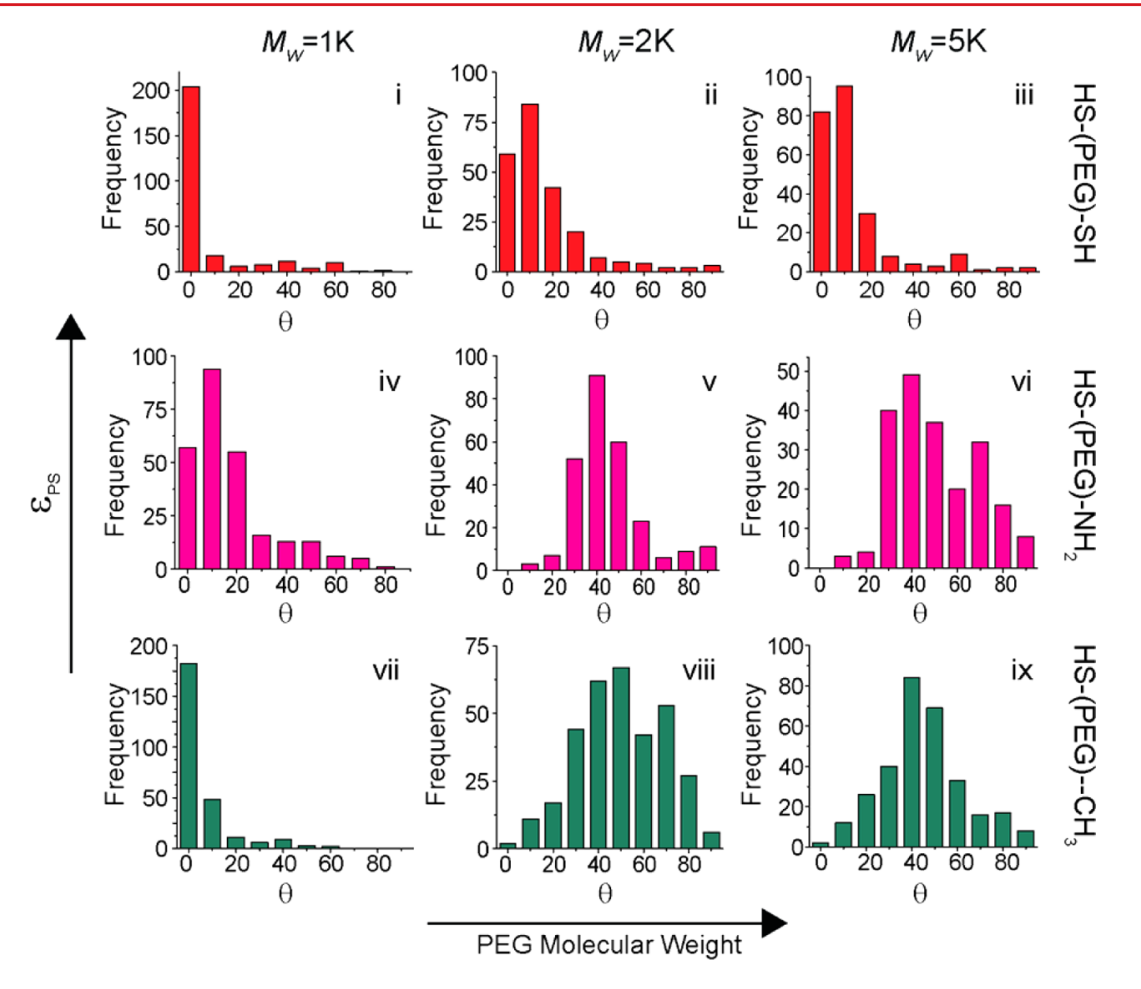

Figure 3. Statistical analysis showing the distribution of nanocube orientation angles in each graft-specific assembly. 

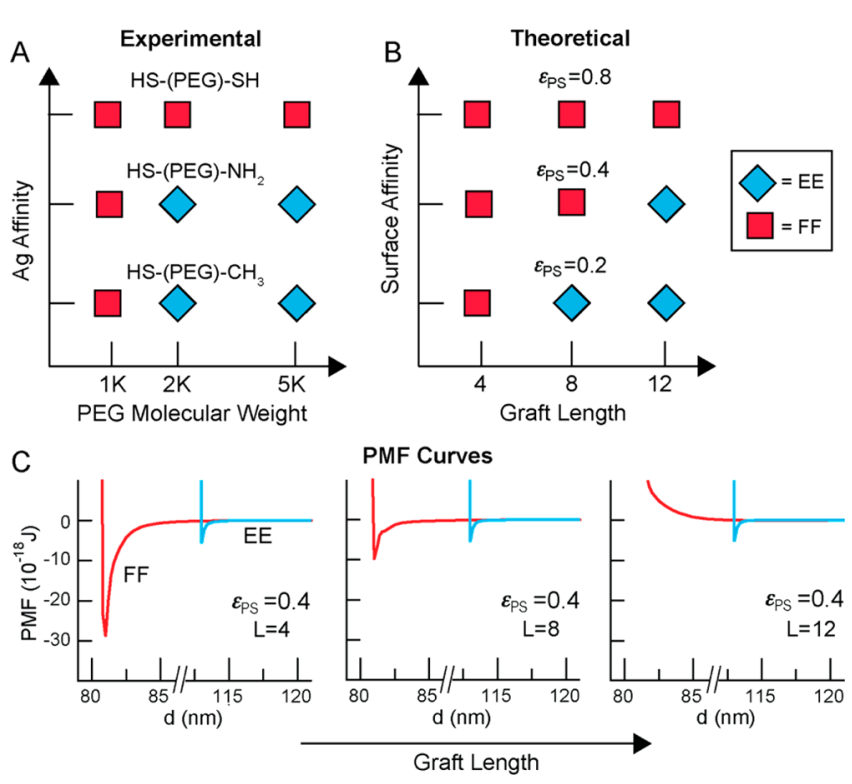

Figure 4. (A) Experimental nanocube orientation phase diagram showing observed orientations of nanocube for varying graft molecular weight and Ag affinity of the end functional group. (B) Theoretical nanocube orientation phase diagram showing observed orientation of nanocubes for varying $\mathrm{L}$ and $\varepsilon_{\mathrm{PS}}$. $(\mathrm{C})$ Potential mean force curves corresponding to the data points in panel (B) for $L=4,8$, and 12 and $\varepsilon_{\mathrm{PS}}=0.4$. The $x$-axis corresponds to the center-to-center distance, $d$, between nanocubes.

We also observe that nanocubes modified with PEG-DT display no critical $L$ within the range of molecular weights that we tested. Even for $\mathrm{M}_{\mathrm{W}}=5 \mathrm{k}$, we observe that the nanocubes mainly adopt FF orientations with narrow nanojunction angle distributions. To better understand this result, we measured the interparticle center-to-center distance for each nanocube assembly in Figure 5. Based on literature values for polymer chains grafted to a solid surface, the total distance contributed from the grafted PEG chains are expected to be 2.2, 5.6, and $11.2 \mathrm{~nm}$ for PEG chains with $\mathrm{M}_{\mathrm{W}}=1 \mathrm{k}, 2 \mathrm{k}$ and $5 \mathrm{k}$, respectively. $^{40-42}$ For nanocubes grafted with $\mathrm{mPEG}$, the interparticle distances are approximately 1.5 times the polymer graft length: $3.2 \pm 1.2 \mathrm{~nm}, 9.4 \pm 1.3 \mathrm{~nm}$, and $18.8 \pm 2.5 \mathrm{~nm}$ for Ag nanocubes modified with $\mathrm{M}_{\mathrm{W}}=1 \mathrm{k}, 2 \mathrm{k}$, and $5 \mathrm{k} \mathrm{mPEG}$, respectively. Nanocubes grafted with short PEG-Am chains with $\mathrm{M}_{\mathrm{W}}=1 \mathrm{k}$ also possess an average interparticle distance of $3.7 \pm 1.9 \mathrm{~nm}$, suggesting significant interdigitation for these FForiented cubes. However, nanocubes grafted with longer PEGAm chains exhibit distances that correspond to approximately double the graft length $\left(14.6 \pm 2 \mathrm{~nm}\right.$ for $\mathrm{M}_{\mathrm{W}}=2 \mathrm{k}$ and $24.4 \pm$ $2.8 \mathrm{~nm}$ for $\mathrm{M}_{\mathrm{W}}=5 \mathrm{k}$ ), suggesting almost no interdigitation occurs between the amine-terminated grafts for neighboring nanocubes. This could be due to hydrogen bonding interactions between the terminal amine groups on neighboring nanocubes, which prevents graft interdigitation. Finally, nanocubes grafted with PEG-DT exhibit the shortest interparticle distances, with $3.2 \pm 1.3,7.4 \pm 1.6$, and $13.2 \pm 1.6 \mathrm{~nm}$ for $\mathrm{Ag}$ nanocubes modified with $\mathrm{M}_{\mathrm{W}}=1 \mathrm{k}, 2 \mathrm{k}$, and 5k PEG-DT, respectively. These interparticle distances correspond to a little over one graft chain length and suggest that terminal thiol groups of the PEG-DT graft may actually bind to the Ag surface of the adjacent nanocube (Figure 5B). This is consistent with the expected large $\varepsilon_{\mathrm{PS}}$ value for the PEG-DT.
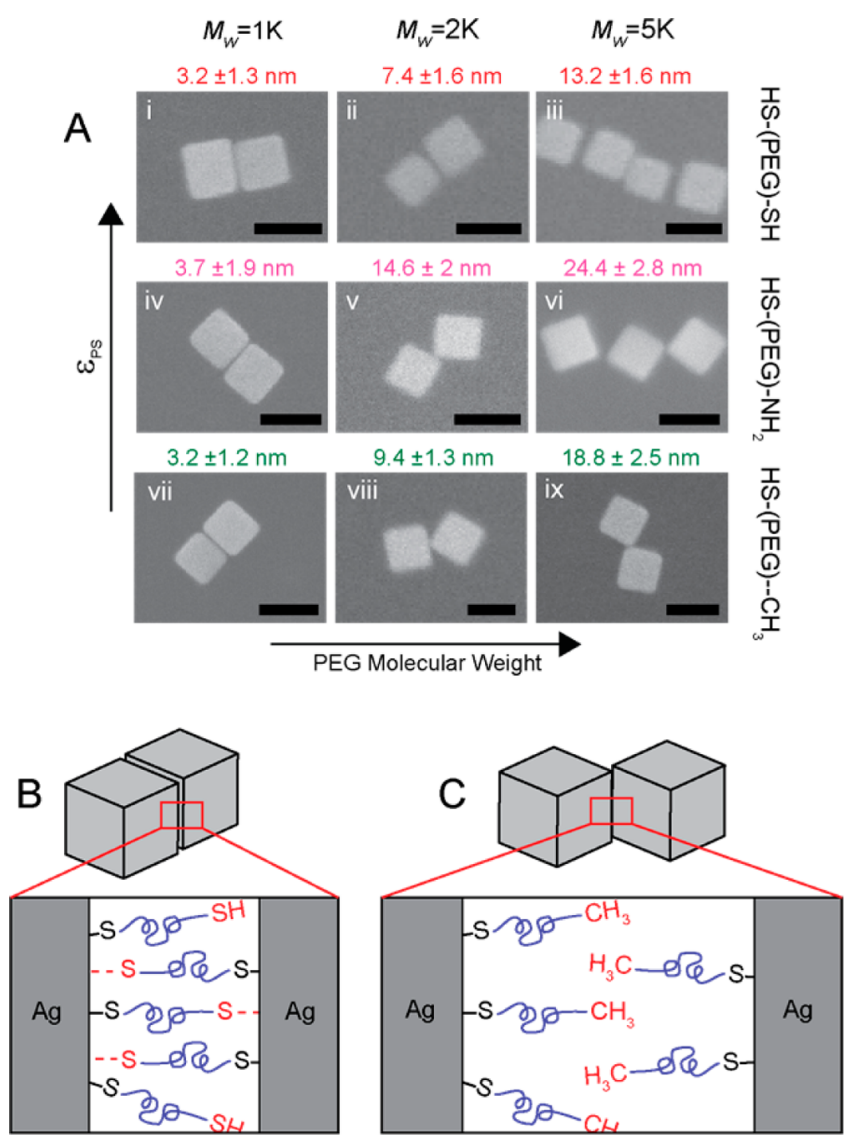

Figure 5. (A) SEM images showing varying interparticle distance in PEG dithiol (i, ii, iii), thiol PEG amine (iv, v, vi), and mPEG thiol (vii, viii, ix) induced assemblies (scale bar $100 \mathrm{~nm}$ ). (B,C) Functional ligand mediated interdigitation by PEG dithiol drives Ag nanocube in FF orientation and weak mPEG thiol in EE orientation.

Conclusion. Our experiments allow us to directly map our assembly results onto our predicted phase diagram for nanocube orientation. Overall, we observe results consistent with our previous theoretical prediction regarding the effect of $\varepsilon_{\mathrm{PS}}$ on nanocube orientation. ${ }^{9}$ The presence of a chemical functionality that is strongly metal-binding produces nanocubes that are FF oriented, regardless of polymer graft length. This is observed for thiol-terminated grafts, which result in a strong $\mathrm{Ag}-\mathrm{S}$ bond, with a dissociation energy of $216.7 \pm 14.6 \mathrm{~kJ} /$ mol. ${ }^{43}$ However, the $\mathrm{Ag}-\mathrm{CH}_{3}$ bond dissociation energy is expected to be $134.1 \pm 6.8 \mathrm{~kJ} / \mathrm{mol}$ and $\mathrm{Ag}-\mathrm{NH}_{2}$ is approximately $191.04 \mathrm{~kJ} / \mathrm{mol}^{44}$ where only short polymer grafts terminated with $-\mathrm{CH}_{3}$ and $-\mathrm{NH}_{2}$ result in $\mathrm{FF}$ orientations. One significant difference between our experiments and our theoretical models is that our experiments were carried out with polymer grafts that possess terminal functional groups, whereas our theoretical results were carried out for nanocubes passivated with homogeneous polymer grafts. In these models, $\varepsilon_{\mathrm{PS}}$ is modified by modulating the interaction potential of every monomer along the graft chain. This discrepancy makes it difficult to gauge the role of the PEG chain in our assembly experiments, including how the PEG itself contributes to $\varepsilon_{\mathrm{PS}}$. However, our results strongly suggest that the identity of the polymer chain itself is likely to not contribute significantly to $\varepsilon_{\mathrm{PS}}$ as long as the $\mathrm{Ag}$-monomer binding strength is relatively low. 


\section{ASSOCIATED CONTENT}

\section{S Supporting Information}

The Supporting Information is available free of charge on the ACS Publications website at DOI: 10.1021/acs.nanolett.5b02748.

Experimental methods, Ag nanocube synthesis, polymer thin film, monolayer deposition, surface modification, annealing conditions, and additional data (PDF)

\section{AUTHOR INFORMATION}

\section{Corresponding Author}

*E-mail: atao@ucsd.edu. Phone: (858) 822-4237. Fax: (858) 534-9533.

\section{Notes}

The authors declare no competing financial interest.

\section{ACKNOWLEDGMENTS}

This work is funded by office of Naval Research (Award No. N000141210574) and a grant from National Science Foundation (CMMI, Award No. 1200850).

\section{REFERENCES}

(1) Anker, J. N.; Hall, W. P.; Lyandres, O.; Shah, N. C.; Zhao, J.; Van Duyne, R. P. Nat. Mater. 2008, 7, 442-453.

(2) Stewart, M. E.; Anderton, C. R.; Thompson, L. B.; Maria, J.; Gray, S. K.; Rogers, J. A.; Nuzzo, R. G. Chem. Rev. 2008, 108, 494521.

(3) Hao, F.; Sonnefraud, Y.; Dorpe, P. V.; Maier, S. A.; Halas, N. J.; Nordlander, P. Nano Lett. 2008, 8, 3983-3988.

(4) Kneipp, K.; Wang, Y.; Kneipp, H.; Perelman, L. T.; Itzkan, I.; Dasari, R. R.; Feld, M. S. Phys. Rev. Lett. 1997, 78, 1667-1670.

(5) Talley, C. E.; Jackson, J. B.; Oubre, C.; Grady, N. K.; Hollars, C. W.; Lane, S. M.; Huser, T. R.; Nordlander, P.; Halas, N. J. Nano Lett. 2005, 5, 1569-1574.

(6) Cai, W.; Brongersma, M. L. Nat. Nanotechnol. 2010, 5, 485-486.

(7) Atwater, H. A.; Polman, A. Nat. Mater. 2010, 9, 205-213.

(8) Zhang, N.; Liu, Y. J.; Yang, J.; Su, X.; Deng, J.; Chum, C. C.; Hong, M.; Teng, J. Nanoscale 2014, 6, 1416-1422.

(9) Gao, B.; Arya, G.; Tao, A. R. Nat. Nanotechnol. 2012, 7, 433-437.

(10) Park, D. J.; Zhang, C.; Ku, J. C.; Zhou, Y.; Schatz, G. C.; Mirkin,

C. A. Proc. Natl. Acad. Sci. U. S. A. 2015, 112, 977-981.

(11) Haji-Akbari, A.; Engel, M.; Glotzer, S. C. J. Chem. Phys. 2011, $135,194101$.

(12) Wang, L.; Xu, L.; Kuang, H.; Xu, C.; Kotov, N. A. Acc. Chem. Res. 2012, 45, 1916-1926.

(13) Sohn, B.-H.; Choi, J.-M.; Yoo, S. I.; Yun, S.-H.; Zin, W.-C.; Jung, J. C.; Kanehara, M.; Hirata, T.; Teranishi, T. J. Am. Chem. Soc. 2003, $125,6368-6369$.

(14) Lin, Y.; Boker, A.; He, J.; Sill, K.; Xiang, H.; Abetz, C.; Li, X.; Wang, J.; Emrick, T.; Long, S.; Wang, Q.; Balazs, A.; Russell, T. P. Nature 2005, 434, 55-59.

(15) Thorkelsson, K.; Mastroianni, A. J.; Ercius, P.; Xu, T. Nano Lett.

2012, 12, 498-504.

(16) Thorkelsson, K.; Nelson, J. H.; Alivisatos, A. P.; Xu, T. Nano Lett. 2013, 13, 4908-4913.

(17) Bedrov, D.; Smith, G. D.; Smith, J. S. J. Chem. Phys. 2003, 119, 10438-10447.

(18) Striolo, A.; Egorov, S. A. J. Chem. Phys. 2007, 126, 014902.

(19) Kim, S.-C.; Lee, S. H. Mol. Phys. 2006, 104, 1487-1495.

(20) Li, Z.; Wu, J. J. Chem. Phys. 2007, 126, 144904.

(21) Jayaraman, A.; Schweizer, K. S. Macromolecules 2008, 41, 94309438

(22) Smith, G. D.; Bedrov, D. Langmuir 2009, 25, 11239-11243.

(23) Hattemer, G. D.; Arya, G. Macromolecules 2015, 48, 1240-1255.
(24) Bozorgui, B.; Sen, M.; Miller, W. L.; Pàmies, J. C.; Cacciuto, A. J. Chem. Phys. 2010, 132, 014901.

(25) Phillips, C. L.; Iacovella, C. R.; Glotzer, S. C. Soft Matter 2010, 6, 1693-1703.

(26) Chen, T.; Zhang, Z.; Glotzer, S. C. Langmuir 2007, 23, 65986605 .

(27) Akcora, P.; Liu, H.; Kumar, S. K.; Moll, J.; Li, Y.; Benicewicz, B. C.; Schadler, L. S.; Acehan, D.; Panagiotopoulos, A. Z.; Pryamitsyn, V.; Ganesan, V.; Ilavsky, J.; Thiyagarajan, P.; Colby, R. H.; Douglas, J. F. Nat. Mater. 2009, 8, 354-359.

(28) Klinkova, A.; Thérien-Aubin, H.; Ahmed, A.; Nykypanchuk, D.; Choueiri, R. M.; Gagnon, B.; Muntyanu, A.; Gang, O.; Walker, G. C.; Kumacheva, E. Nano Lett. 2014, 14, 6314-6321.

(29) Murthy, C. R.; Gao, B.; Tao, A. R.; Arya, G. Nanoscale 2015, 7, 9793-9805.

(30) Stewart, A.; Zheng, S.; McCourt, M. R.; Bell, S. E. J. ACS Nano 2012, 6, 3718-3726.

(31) Das, T.; Kumar, A.; Ghosh, P.; Maity, A.; Jaffer, S. S.; Purkayastha, P. J. Phys. Chem. C 2010, 114, 19635-19640.

(32) Kemp, M. M.; Kumar, A.; Mousa, S.; Dyskin, E.; Yalcin, M.; Ajayan, P.; Linhardt, R. J.; Mousa, S. A. Nanotechnology 2009, 20, 455104.

(33) Djaoued, Y.; Badilescu, S.; Balaji, S.; Seirafianpour, N.; Hajiaboli, A. R.; Banan Sadeghian, R.; Braedley, K.; Brüning, R.; Kahrizi, M.; Truong, V. V. Appl. Spectrosc. 2007, 61, 1202-1210.

(34) Anema, J. R.; Brolo, A. G.; Felten, A.; Bittencourt, C. J. Raman Spectrosc. 2010, 41, 745-751.

(35) Moran, C. H.; Rycenga, M.; Zhang, Q.; Xia, Y. J. Phys. Chem. C 2011, 115, 21852-21857.

(36) Koenig, J. L.; Angood, A. C. Journal of Polymer Science Part A-2: Polym. Phys. 1970, 8, 1787-1796.

(37) Krishnan, K.; Krishnan, R. S. Proc. Indian Acad. Sci. A 1966, 64, $111-122$.

(38) Joo, S. W.; Han, S. W.; Kim, K. Langmuir 2000, 16, 5391-5396.

(39) Lin-Vien, D.; Colthup, N. B.; Fateley, W. G.; Grasselli, J. G. The

Handbook of Infrared and Raman Characteristic Frequencies of Organic Molecules; Academic Press: San Diego, CA, 1991.

(40) Zhu, B.; Eurell, T.; Gunawan, R.; Leckband, D. J. Biomed. Mater. Res. 2001, 56, 406-16.

(41) Fang, Y.; Xu, W.; Wu, J.; Xu, Z. K. Chem. Commun. 2012, 48, $11208-11210$.

(42) Levin, C. S.; Bishnoi, S. W.; Grady, N. K.; Halas, N. J. Anal. Chem. 2006, 78, 3277-3281.

(43) Luo, Y. R. Handbook of Bond Dissociation Energies in Organic Compounds; CRC Press: Boca Raton, FL, 2003.

(44) Jung, I.; Shin, K.; Kim, N. R.; Lee, H. M. J. Mater. Chem. C 2013, 1, 1855-1862. 